\section{Note added in proof}

Since submission of this paper, Roberts and Duckett (1978) have reported a live born female infant with trisomy $16 \mathrm{p}$. They also reviewed published reports. Their report modifies the opinion given in the last paragraph of this paper in which it is proposed that triplication of the short arm of chromosome 16 may be incompatible with live birth and may be responsible for fetal loss in full trisomy 16.

\section{Reference}

Roberts, S. H., and Duckett, D. P., (1978). Trisomy 16p in a liveborn infant and a review of partial and full trisomy 16. Journal of Medical Genetics, 15, 375-381.

\section{Two children with partial trisomy for $7 \mathrm{p}$}

SUMMARY A second family in which a balanced translocation between $7 p$ and $22 q$ is segregating is described. The clinical features of 2 children with a resulting partial trisomy for $7 p$ are described and compared with the previously described case.

The report by Larson et al. (1977) of a family in which a $7 p ; 22 q$ translocation was segregating prompts us to report a similar family with 2 further children with the unbalanced karyotype and partial $7 p$ trisomy.

\section{Case reports}

CASE 1

This case (PRU 3373) was a baby girl born in 1966 after a normal pregnancy of $\mathbf{4 0}$ weeks' gestation, birthweight $2 \cdot 30 \mathrm{~kg}$. She was hypotonic with a weak cry and multiple anomalies were noted at birth: the skull was elongated with a widely separated metopic suture; she had microphthalmos, the eyes having a mongoloid slant; ears were low set and there was micrognathia; a systolic murmur was present. There were a number of skeletal abnormalities which included arachnodactyly, flexion deformity of the wrists, talipes calcaneo-valgus, and widely separated 1st and 2nd toes. A single palmar crease was present unilaterally. She failed to thrive and died aged 8 weeks.

At necropsy, in addition to the features noted above, she had hydrocephalus and microgyria, a hypertrophic left ventricle with persistent ductus arteriosus, and some aortic stenosis proximal to the ductus. Both kidneys were small with cysts.
Chromosome analysis in 1966 using Orceiß stain showed a chromosome complement of? $46, \mathrm{XX}, \mathrm{Gq}+$.

\section{CASE 2}

This case (PRU 3373), the brother of case 1, was bor in 1968 at 38 weeks' gestation, birthweight $3.94 \mathrm{~kg}$. His mother had had influenza at the beginning of the pregnancy. Delivery was in breech presentation and a left brachial plexus lesion was noted almosf immediately. He looked similar to his sister, case 1.0 When seen at the age of 6 months (by Dr S. M $\overrightarrow{\dot{\vec{\omega}}}$ Kohlinsky), his head circumference was $44 \mathrm{cn}$ $(+1$ SD) and the occiput was prominent. He had $\bar{\Phi}$ long face with narrow palpebral fissures, especially on the left, with a slight mongoloid slant to the eye and epicanthic folds. The nasal bridge was broad and flat, the palate was high and arched, and the् maxilla prominent with a thick lower lip. Sligh weakness of the left arm and shoulder was present? The cardiovascular, respiratory, and genitourinar $\vec{x}$ systems appeared normal. Developmental leve告 was $2 \frac{1}{2}$ to 3 months.

Because of his coarse facies, his urinary muco polysaccharides were measured and found to be normal. Peripheral blood chromosome analysis using Orcein stain showed a chromosome complee ment of $46, \mathrm{XY}, \mathrm{Gq}+$, similar to his sister, case 1 . ¿

$\mathrm{He}$ has remained extremely retarded and at the age of 9 years can sit unaided but cannot stand of walk. He says no words and is unable to feed him self. He has had one fit, but is not currently on medication.

\section{Family studies}

The mother (born in 1936) has rheumatoid arthritis the father (born in 1926) is healthy and they are na blood relations. The mother had 2 miscarriages, one at 14 and the other at 16 weeks' gestation, but healthy sons were born in 1970 and in 1973 . In 196\% peripheral blood chromosome analysis using Orcei stain showed the father to have a chromosome complement of $46, \mathrm{XY}, \mathrm{Gq}+$ and in 1970 one of the healthy sons was found to have this karyotyp also. The father was an only child and no furthe family studies have been attempted.

It seemed most likely that a balanced translocatiofy was involved, but it was impossible to distinguist the karyotypes of the phenotypically normal family members from those of the abnormal before the development of banding techniques. Early attemp每 at banding in 1973 were unsatisfactory and for the reason the family decided against prenatal chromor some analysis during the final pregnancy. Chromos some analysis after birth showed the infant to hav a normal male chromosome complement $46, X Y$. 


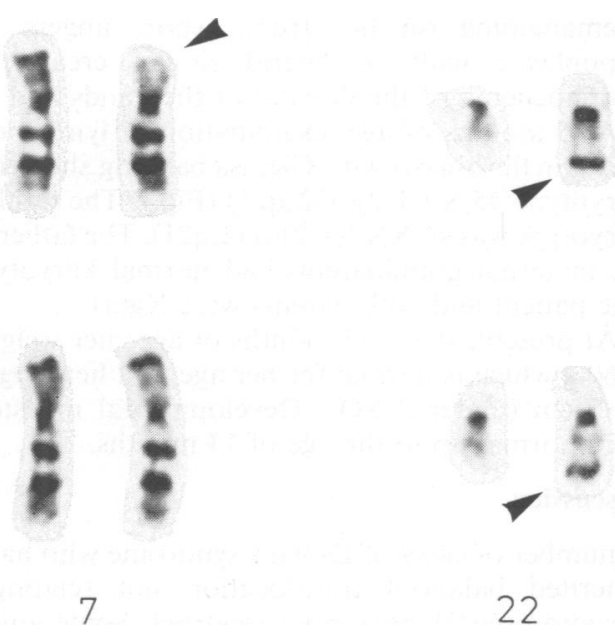

Fig. (a) Partial karyotype of father showing balanced reciprocal translocation; (b) partial karyotype of case 2 showing partial trisomy for $7 p$.

In 1974, re-evaluation of the family using Gbanding identified the translocation. Balanced family members had chromosome complements 46,XY,t(7;22)(p15;q13) (Fig. a). Case 2 had the unbalanced karyotype $46, \mathrm{XY}, \operatorname{der}(22), \mathrm{t}(7 ; 22)(\mathrm{p} 15$; q13)pat (Fig. b) and therefore had a duplication (partial trisomy) of the distal part of 7p. Case 1 can be assumed to have had the same unbalanced karyotype, since she had the derived chromosome 22.

\section{Discussion}

The only other case of $7 p$ duplication (partial trisomy) of which we are aware is that described by Larson et al. (1977), and there are strong similarities between the two families. In both, the unbalanced situation arose from a reciprocal translocation involving 22q, and in both it was paternal in origin, though, in general, derivative chromosomes are more usually maternally transmitted. Breakpoints in the two families are not identical, 7p21 in Larson's family, and $7 \mathrm{p} 15$ in this family.

The clinical features have certain similarities. Profound motor and mental retardation is the major problem and the necropsy study on case 1 showed a degree of hydrocephalus and microgyria, while an EMI scan on Larson's case showed hypoplasia and atrophy of the brain.

Other common features, such as epicanthic folds and a high arched palate, are non-specific, and our case 1 had skeletal abnormalities not noted in the other two patients. Thus, the most marked effect of the trisomy for $7 p$ seems to be a severe failure in cerebral development resulting in gross retardation, more severe than that found in many other autosomal aneuploidies. In the family described by Larson, however, three unstudied adults were mentally retarded, and therefore thought to have the duplication, though in these cases the effect seemed less severe.

Children with deletions of $7 p$ have been described (reviewed by McPherson et al., 1976) and the most striking clinical feature is craniosynostosis, though the breakpoints appear to be the same as in the present family. Thus, there is no evidence of type and contratype for abnormalities of this chromosome arm.

We thank Professor P. E. Polani for encouragement to publish this report, and acknowledge financial support from the Department of Health and Social Security and the Spastics Society.

A. Caroline Berry, J. Honeycombe, AND S. J. R. MACOUN Paediatric Research Unit, Prince Philip Research Laboratories, Guy's Hospital, London; and St. Luke's Hospital, Guildford, Surrey

\section{References}

Larson, L. M., Wasdahl, W. A., and Jalal, S. M. (1977). Partial trisomy $7 p$ associated with familial $7 p ; 22 q$ translocation. Journal of Medical Genetics, 14, 258-261.

McPherson, E., Hall, J. G., Hickman, R., Gong, B. T., Norwood, T. H., and Hoehn, H. (1976). Chromosome 7 short arm deletion and craniosynostosis. A 7p - syndrome. Human Genetics, 35, 117-123.

Requests for reprints to Dr C. Berry, Paediatric Research Unit, Guy's Hospital Medical School, Guy's Tower, London SE1 9RT.

\section{A case of Turner's syndrome with familial balanced translocation $\mathrm{t}(1 ; 2)(\mathrm{q} 32 ; \mathrm{q} 21) \mathrm{mat}$}

SUMMARY The first case of Turner's syndrome with the familial translocation not involving the $\mathrm{X}$ chromosome is described. The patient had a number of clinical signs of Turner's syndrome and her karyotype was $45, \mathrm{X}, \mathrm{t}(1 ; 2)(\mathrm{q} 32 ; \mathrm{q} 21)$ mat. Though it is speculated that the altered structure of a chromosome may influence meiotic disjunction of a non-homologous chromosome, our case suggests that there may be no relationship between the two chromosomal abnormalities. 